\title{
Remarks on effective action and entanglement entropy of Maxwell field in generic gauge
}

\author{
Sergey N. Solodukhin \\ Laboratoire de Mathématiques et Physique Théorique, \\ Université François-Rabelais Tours Fédération Denis Poisson - CNRS, \\ Parc de Grandmont, 37200 Tours, France \\ KITP, University of California, \\ Santa Barbara, CA 93106, U.S.A. \\ E-mail: Sergey.Solodukhin@lmpt.univ-tours.fr
}

ABSTRACT: We analyze the dependence of the effective action and the entanglement entropy in the Maxwell theory on the gauge fixing parameter $a$ in $d$ dimensions. For a generic value of $a$ the corresponding vector operator is nonminimal. The operator can be diagonalized in terms of the transverse and longitudinal modes. Using this factorization we obtain an expression for the heat kernel coefficients of the nonminimal operator in terms of the coefficients of two minimal Beltrami-Laplace operators acting on 0- and 1-forms. This expression agrees with an earlier result by Gilkey et al. Working in a regularization scheme with the dimensionful UV regulators we introduce three different regulators: for transverse, longitudinal and ghost modes, respectively. We then show that the effective action and the entanglement entropy do not depend on the gauge fixing parameter $a$ provided the certain ( $a$-dependent) relations are imposed on the regulators. Comparing the entanglement entropy with the black hole entropy expressed in terms of the induced Newton's constant we conclude that their difference, the so-called Kabat's contact term, does not depend on the gauge fixing parameter $a$. We consider this as an indication of gauge invariance of the contact term.

KeYwords: Black Holes, Renormalization Regularization and Renormalons

ArXiv EPRINT: 1209.2677 


\section{Contents}

1 Introduction 1

2 Maxwell theory, gauge fixing and nonminimal operator 3

3 Projectors and decomposition of nonminimal operator 4

4 Heat kernel and small $s$ expansion $\quad 5$

$\begin{array}{lll}5 & \text { A formal demonstration of gauge independence } & 6\end{array}$

$\begin{array}{lll}6 & \text { Effective action and UV regulators, gauge independence } & 7\end{array}$

7 Cosmological and Newton's constants $\quad 8$

8 Minkowski spacetime: heat kernel in momentum space $\quad 8$

9 Entanglement entropy $\quad 9$

$\begin{array}{ll}10 \text { Kabat's contact term } & 10\end{array}$

11 Conclusions $\quad 12$

\section{Introduction}

In Quantum Field Theory there are two well-defined quantities that are sensitive to the UV behavior of the theory. One of them is the effective action (for a nice and informative review see [1]) and the other is the entanglement entropy (the various approaches are recently reviewed in $[2-5]$ ). Provided a regularization scheme with a regularization parameter $\epsilon$ is used to handle the UV divergences the both quantities are crucially dependent on the parameter $\epsilon$ thus revealing in this dependence the short-distance behavior of the theory. In the case of the effective action there exist some regularization schemes in which the regularization parameter is dimensionless. The divergences of the action when $\epsilon$ is taken to zero then correspond to the logarithmic UV divergences in those schemes, where a dimensionful $\epsilon$ is introduced. Otherwise, with a dimensionful $\epsilon$, the effective action shows a series of the power-law UV divergent terms. The existing tradition tends to view the power-law UV divergences as spurious and less important than the logarithmic ones, the latter are considered to be "physical" while the former are viewed as "scheme dependent". This is especially astonishing taking that namely a power law UV divergent term in the effective action produces that enormous contribution to the cosmological constant which leads to the well known "cosmological constant problem". 
The situation with the entanglement entropy is quite the opposite. Here the leading and the most important contribution to the entropy is given by the area of the entangling surface. So that a dimensionful UV regulator is natural to be present in order to, in a combination with the area, produce a dimensionless quantity. Thus, in this case the power-law UV divergences are viewed as physically important.

The different attitude towards the power law UV divergences demonstrated in these two cases is surprising since, for all fields minimally coupled to gravity, there is a correspondence between the UV divergences in the effective action and the entropy and, if we are talking about the entanglement entropy of horizons, the renormalization of one quantity automatically leads to the renormalization of the other [6, 7]. Moreover, by changing the shortdistance behavior of the theory one can see that the both quantities demonstrate, in parallel, the corresponding modifications in the structure of the UV divergences [8]. The whole issue of the power law UV terms in the effective action becomes especially important in the models of induced gravity, where both the cosmological and Newton's constants and the black hole entropy are induced in quantum loops of some fundamental constituents $[9,10]$.

By taking seriously the power-law UV divergences in the effective action we, however, may have a situation, where some self-obvious properties of the effective action are less transparent. In particular, the usual demonstration of gauge invariance of the effective action should be re-derived ${ }^{1}$ in this case and, possibly, supplemented by imposing some additional requirements.

In the present paper we consider these issues in the case of the Maxwell theory. Following the standard gauge fixing procedure, we add a generic gauge fixing term to the action. Our goal then is to demonstrate that the resultant effective action and the entropy do not actually depend on the value of the gauge fixing parameter $a$. This is not an obvious property of the theory since, for generic values of the gauge fixing parameter, $a \neq 1$, the corresponding vector field operator is nonminimal. The separation of vector modes on the transverse and longitudinal helps to diagonalize the operator and effectively reduce the problem to the one in the minimal gauge $a=1$. That the effective action and the entanglement entropy do not depend on parameter $a$, however, is not achieved automatically and requires us to impose some additional conditions on the regularization parameters which regularize the UV divergences in each sector: transverse, longitudinal and ghost. That one has to introduce different UV regulators for the transverse and longitudinal modes was earlier proposed by Kabat [12] in two dimensions. The relations which we derive for the UV regulators in the different sectors of the theory are consistent with those proposed in [12]. The Maxwell field can be embedded into various supersymmetric multiplets for which the entropy has been recently analyzed in [13-16]. We believe our analysis should be important for this class of theories too.

\footnotetext{
${ }^{1}$ In particular, the use of Ward's identities may fail for the power-law divergent terms in the effective action $[11]$.
} 


\section{Maxwell theory, gauge fixing and nonminimal operator}

We start with the standard action for the Maxwell theory with a generic gauge fixing term

$$
W=\int d^{x} \sqrt{g}\left(\frac{1}{4} F_{\mu \nu} F^{\mu \nu}+\frac{a}{2}\left(\nabla_{\mu} A^{\mu}\right)^{2}\right) .
$$

The corresponding field equation for the vector field $A_{\mu}$,

$$
\Delta_{(a) \nu}^{\mu} A^{\nu}=0
$$

is governed by a nonminimal operator

$$
\Delta_{(a) \nu}^{\mu}=-\square_{1} \delta_{\nu}^{\mu}+(1-a) \nabla^{\mu} \nabla_{\nu}+R_{\nu}^{\mu}
$$

We define $\square_{k}=\nabla_{\alpha} \nabla^{\alpha}$ as the Laplace operator acting on $k$-forms. The operator (2.3) becomes minimal if $a=1$. Below we will consider the general case, when $a$ is arbitrary ${ }^{2}$ parameter. It should be note that in the coordinate invariant form the non-minimal operator (2.3) can be represented as follows

$$
\Delta_{a}=-\left(\delta_{2} d_{1}+a d_{0} \delta_{1}\right)
$$

where $d_{k}$ is the differential exterior operator acting on $k$-forms and $\delta_{k+1}$ is its adjoint operator acting on $(k+1)$-forms with the well known properties $d_{k} d_{(k-1)}=0$ and $\delta_{(k+1)} \delta_{k}=0$. If $a=1$ then $\Delta_{1}=-\left(d_{0} \delta_{1}+\delta_{2} d_{1}\right)$ is the Beltrami-Laplace operator on 1-forms,

$$
\Delta_{1 \nu}^{\mu}=-\square_{1} \delta_{\nu}^{\mu}+R_{\nu}^{\mu}
$$

The action (2.1) should be supplemented by a ghost action

$$
W_{g h}=\int d^{d} x \sqrt{g} \frac{1}{2} \bar{c} \Delta_{g h} c
$$

where the ghost operator is

$$
\Delta_{g h}=-\sqrt{a} \square_{0}
$$

We note that a factor $\sqrt{a}$ here is related to the Nielsen-Kallosh determinant coming from the path integral of the auxilliary scalar parameter used to justify the t'Hooft trick. So that the quantum partition function, provided the generic gauge is imposed, of the theory reads

$$
Z(a)=\operatorname{det}^{-1 / 2} \Delta_{(a)} \operatorname{det} \Delta_{g h} .
$$

In our analysis of how (2.8) depends on the gauge fixing parameter $a$ it will be, however, useful to separate the contributions of the transverse and longitudinal modes in the partition function (2.8).

\footnotetext{
${ }^{2}$ The unitarity requires that $a$ to be positive. So no any other restriction will be imposed on $a$.
} 


\section{Projectors and decomposition of nonminimal operator}

Let us introduce the longitudinal and transverse mutually orthogonal projectors

$$
\begin{aligned}
\mathcal{P}^{\mu}{ }_{\nu} & =\nabla^{\mu} \frac{1}{\square_{0}} \nabla_{\nu}, & \Pi^{\mu}{ }_{\nu}=\delta^{\mu}{ }_{\nu}-\mathcal{P}^{\mu}{ }_{\nu} & \\
\mathcal{P} \mathcal{P} & =\mathcal{P}, & \Pi \Pi=\Pi, & \mathcal{P} \Pi=\Pi \mathcal{P}=0,
\end{aligned}
$$

where $\square_{0}$ is the covariant Laplace operator acting on scalars (0-form), $\square_{0}=\nabla_{\alpha} \nabla^{\alpha}$. They act on vector field $A^{\nu}$ as $(\mathcal{P} A)^{\mu}=\mathcal{P}_{\nu}^{\mu} A^{\nu}$. Similarly, we can consider the Ricci tensor $R_{\nu}^{\mu}$ as operator acting on vector fields, $(R A)^{\mu}=R_{\nu}^{\mu} A^{\nu}$. Clearly, the operator $\Pi$ is projector onto transverse vector fields, $\nabla_{\mu}\left(\Pi_{\nu}^{\mu} A^{\nu}\right)=0$.

We note the useful commutation relations between the covariant Laplace operator $\square_{k}$ and the covariant derivative

$$
\begin{aligned}
& \nabla^{\mu} \square_{0}=\square_{1} \nabla^{\mu}-R^{\mu}{ }_{\nu} \nabla^{\nu}, \\
& \nabla_{\mu} \square_{1}=\square_{0} \nabla_{\mu}+\nabla_{\alpha} R_{\mu}^{\alpha},
\end{aligned}
$$

where in the first line the operators are acting on scalars while in the second they are acting on vectors and the Ricci tensor is viewed as a matrix operator. Using these relations we find

$$
\nabla^{\mu} \nabla_{\nu}=\square_{1} \mathcal{P}_{\nu}^{\mu}-R_{\alpha}^{\mu} \mathcal{P}_{\mu}^{\alpha}
$$

So that the non-minimal operator (2.3) can be presented in the form

$$
\Delta_{(a) \nu}^{\mu}=\Delta_{1 \alpha}^{\mu} \Pi_{\nu}^{\alpha}+a \Delta_{1 \alpha}^{\mu} \mathcal{P}_{\nu}^{\alpha},
$$

where $\Delta_{1}$ is the minimal operator (2.5). It is now natural to define the transverse and longitudinal Laplace operators

$$
\Delta_{T}=\Delta_{1} \Pi, \Delta_{L}=\Delta_{1} \mathcal{P}
$$

so that we have

$$
\Delta_{a}=\Delta_{T}+a \Delta_{L}
$$

In terms of the covariant differential operators $d$ and $\delta$ the projector $\mathcal{P}$ takes the form

$$
\mathcal{P}=d_{0} \frac{1}{\square_{0}} \delta_{1},
$$

we remind that $\square_{0}=\delta_{1} d_{0}$. Using this representation and the invariant form (2.4) of the operator $\Delta_{a}$ we find that the operators $\Delta_{T}$ and $\Delta_{L}$ are, in fact, local,

$$
\Delta_{T}=-\delta_{2} d_{1}, \Delta_{L}=-d_{0} \delta_{1} .
$$

Using these invariant representations it is easy to demonstrate the commutation relations

$$
\left[\Delta_{1}, \mathcal{P}\right]=0,\left[\Delta_{T}, \Delta_{L}\right]=0
$$


Moreover, since $\mathcal{P}$ commutes with operator $\Delta_{1}$ and that $\mathcal{P}$ and $\Pi$ are orthogonal then the product of any powers of operators $\Delta_{T}$ and $\Delta_{L}$ is nul,

$$
\Delta_{L}^{k} \cdot \Delta_{T}^{n}=0, k, n>0 .
$$

These properties indicate that the determinant of the operator $\Delta_{a}$, defined as product of its non-zero eigen values, reduces to a product of two determinants

$$
\operatorname{det} \Delta_{a}=\operatorname{det} \Delta_{T} \operatorname{det}\left(a \Delta_{L}\right) \text {. }
$$

This representation should be used in (2.8) when we compute the partition function of the Maxwell field.

\section{$4 \quad$ Heat kernel and small $s$ expansion}

The technical tool to be used in this paper in order to evaluate the partition function (2.8) is the heat kernel. For a vector operator $\mathcal{D}^{\mu}{ }_{\nu}$ the heat kernel $K_{\nu}^{\mu}\left(s, x, x^{\prime}\right)$ satisfies the equation

$$
\partial_{s} K_{\nu}^{\mu}\left(s, x, x^{\prime}\right)+\mathcal{D}_{\sigma}^{\mu}(x) K_{\nu}^{\sigma}\left(s, x, x^{\prime}\right)=0
$$

and the "initial" condition

$$
K_{\nu}^{\mu}\left(s=0, x, x^{\prime}\right)=\delta_{\nu}^{\mu} \delta^{(d)}\left(x, x^{\prime}\right) .
$$

With the help of the heat kernel we can express the effective action $W_{\text {eff }}=-\frac{1}{2} \ln \operatorname{det} \mathcal{D}$ as follows

$$
W_{\mathrm{eff}}=-\frac{1}{2} \int_{\epsilon^{2}}^{\infty} \frac{d s}{s} \int d^{d} x \operatorname{Tr} K\left(s, x^{\prime}=x\right),
$$

where $\epsilon$ is an UV cut-off. The heat kernel is characterized by its small $s$ expansion,

$$
\operatorname{Tr} K\left(s, x=x^{\prime}\right)=\frac{1}{(4 \pi)^{d / 2}} \sum_{k=0} c_{k}(\mathcal{D}) s^{\frac{2 k-d}{2}} .
$$

A formal solution to equation (4.1) is $K=e^{-s \mathcal{D}}$. For the nonminimal operator $\Delta_{a}$ the heat kernel reduces, as can be seen by using properties (3.9) and (3.10), to a sum of two heat kernels

$$
\begin{aligned}
e^{-s \Delta_{a}} & =e^{-s \Delta_{T}}+e^{-s a \Delta_{L}} \\
& =e^{-s \Delta_{1}} \Pi+e^{-s a \Delta_{1}} \mathcal{P} .
\end{aligned}
$$

This relation can be obtained by first using the representation of exponential $e^{-s \Delta_{a}}$ as Taylor series and then using the commutation relations (3.9) and the orthogonality property (3.10). Consider now a small $s$ expansion of the heat kernels in (4.5). We find a relation

$$
c_{k}\left(\Delta_{a}\right)=c_{k}\left(\Delta_{T}\right)+a^{\frac{2 k-d}{2}} c_{k}\left(\Delta_{L}\right) .
$$

Operator $\Delta_{L}=-d_{0} \delta_{1}$ has same non-zero eigen values as the scalar Laplace operator $-\square_{0}=-\delta_{1} d_{0}$, hence one has that $c_{k}\left(\Delta_{L}\right)=c_{k}\left(-\square_{0}\right)$. On the other hand, if $a=1$ then 
$c_{k}\left(\Delta_{1}\right)=c_{k}\left(\Delta_{T}\right)+c_{k}\left(\Delta_{L}\right)$ from which we find that $c_{k}\left(\Delta_{T}\right)=c_{k}\left(\Delta_{1}\right)-c_{k}\left(-\square_{0}\right)$. Putting everything together we express the heat kernel coefficients of the non-minimal operator $\Delta_{a}$ in terms of the coefficients of the heat kernel of two minimal operators,

$$
c_{k}\left(\Delta_{a}\right)=c_{k}\left(\Delta_{1}\right)+\left(a^{\frac{2 k-d}{2}}-1\right) c_{k}\left(-\square_{0}\right) .
$$

This relation agrees with an earlier result obtained in [17] (see also [18] and [1] for a related discussion). We remind the reader the first few heat kernel coefficients of the minimal operators,

$$
\begin{aligned}
c_{0}\left(-\square_{0}\right) & =\sqrt{g}, c_{1}\left(-\square_{0}\right)=\sqrt{g} \frac{1}{6} R, \\
c_{0}\left(\Delta_{1}\right) & =\sqrt{g} d, c_{1}\left(\Delta_{1}\right)=\sqrt{g}\left(\frac{d}{6} R-R\right) .
\end{aligned}
$$

We have independently checked the relation (4.7) for coefficients with $k=0,1$ by using the momentum space method developed in [8] (details of this calculation are not included here and are available upon request) and we have obtained a complete agreement with (4.7).

\section{A formal demonstration of gauge independence}

Let us start with a formal demonstration that the partition function (2.8) does not depend on the gauge fixing parameter $a$. Our starting point is the variation formula for the determinant. This formula can be obtained by varying the identity $\ln \operatorname{det} \mathcal{D}=\operatorname{Tr} \ln \mathcal{D}$, valid for any operator $\mathcal{D}$,

$$
\delta \ln \operatorname{det} \mathcal{D}=\operatorname{Tr} \mathcal{D}^{-1} \delta \mathcal{D},
$$

where $\mathcal{D}^{-1}$ is the inverse operator. It can be represented by integral over proper time

$$
\mathcal{D}^{-1}=\int_{\epsilon^{2}}^{\infty} d s e^{-s \mathcal{D}}
$$

where we introduced a regulator $\epsilon$. In what follows we introduce a separate regulator for each operator in question: $\epsilon_{T}, \epsilon_{L}$ and $\epsilon_{G}$.

For the operators at hand we have the following variation with respect to parameter $a$,

$$
\delta \Delta_{a}=\delta a\left(-d_{0} \delta_{1}\right), \quad \delta \Delta_{g h}=\frac{\delta a}{2 \sqrt{a}}\left(-\square_{0}\right) .
$$

For the ghost operator we find

$$
\operatorname{Tr} \Delta_{g h}^{-1} \delta \Delta_{g h}=-\frac{\delta a}{2 \sqrt{a}} \int_{\epsilon_{G}^{2}}^{\infty} d s \operatorname{Tr}\left(e^{-s \Delta_{g h}} \square_{0}\right)=-\frac{\delta a}{2 a} \int_{\epsilon_{G}^{2} \sqrt{a}}^{\infty} d s \operatorname{Tr}\left(e^{s \square_{0}} \square_{0}\right) .
$$

Then, using that $e^{-s \Delta_{a}} d_{0} \delta_{1}=\left(e^{s \delta_{2} d_{1}}+e^{a s d_{0} \delta_{1}}\right) d_{0} \delta_{1}=e^{a s d_{0} \delta_{1}} d_{0} \delta_{1}$, we obtain for the vector non-minimal operator that

$$
\operatorname{Tr} \Delta_{a}^{-1} \delta \Delta_{a}=-\delta a \int_{\epsilon_{L}^{2}}^{\infty} d s \operatorname{Tr}\left(e^{a s d_{0} \delta_{1}} d_{0} \delta_{1}\right)=-\frac{\delta a}{a} \int_{\epsilon_{L}^{2} a}^{\infty} d s \operatorname{Tr}\left(e^{s \square_{0}} \square_{0}\right),
$$


where we introduced a separate regulator for the longitudinal operator, rescaled the proper time and, finally, used the cyclicity property of the trace, $\operatorname{Tr}\left(d_{0} \delta_{1}\right)^{n}=\operatorname{Tr}\left(\delta_{1} d_{0}\right)^{n}$ and

$$
\operatorname{Tr}\left(e^{s d_{0} \delta_{1}} d_{0} \delta_{1}\right)=\operatorname{Tr}\left(e^{s \delta_{1} d_{0}} \delta_{0} d_{1}\right)=\operatorname{Tr}\left(e^{s \square_{0}} \square_{0}\right) .
$$

In a even more formal analysis one could replace the lower limit in the integrals (5.4), (5.5) by zero. Then one would immediately conclude that the two variations (5.4) and (5.5) mutually cancel each other in the variation of the partition function (2.8). This is that sort of reasoning which is usually used to demonstrate the gauge independence of the partition function.

In the presence of the UV regulators the cancellation of two contributions in the variation of the partition function (2.8) with respect to $a$ is still possible if we impose a certain relation between two regulators,

$$
\epsilon_{G}^{2}=\epsilon_{L}^{2} \sqrt{a} .
$$

This, still rather formal, demonstration can not be correct if the power law UV divergences are present, as we discuss below. A possible reason for this, mentioned to us by A. Barvinsky [11], is the failure of the cyclicity property of trace, used in (5.6), in the presence of the power law UV divergent terms.

\section{Effective action and UV regulators, gauge independence}

Let us now return to the heat kernel representation of the total effective action of the Maxwell theory and separate the three different contributions: transverse, longitudinal and ghost,

$$
W_{\text {Maxwell }}=-\frac{1}{2}\left(\int_{\epsilon_{T}^{2}}^{\infty} \frac{d s}{s} \operatorname{Tr} e^{-s \Delta_{T}}+\int_{\epsilon_{L}^{2}}^{\infty} \frac{d s}{s} \operatorname{Tr} e^{-s a \Delta_{L}}-2 \int_{\epsilon_{G}^{2}}^{\infty} \frac{d s}{s} \operatorname{Tr} e^{-s \Delta_{g h}}\right) .
$$

We remind that the ghost operator $\Delta_{g h}=-\sqrt{a} \square_{0}$.

The relation (5.7) guarantees that dependence on $a$ is not present in the logarithmic terms, proportional to $\ln \epsilon_{L}$ and $\ln \epsilon_{G}$, and in the UV finite terms. If, however, there is a power law divergence of the type $1 / \epsilon^{n}$ in ln det of operators $\Delta_{g h}$ and $\Delta_{L}$ then the relation (5.7) is not enough to guarantee that a power law dependence on $a$ in these terms is absent. This is due to the fact that these terms come with a relative coefficient $-1 / 2$ in (6.1). This relative coefficient is important for the cancellation in the logarithmic terms but does not help at all in the case of the power law divergent terms. Thus, the idea, which is behind the formal analysis in the previous section, that the dependence on the parameter a may disappear due to the mutual cancellation of contributions of the longitudinal and the ghost parts does not work. The only possibility then is that each of the three terms in (6.1), individually, should not depend on $a$.

The important point here is that we introduced three different UV regulators, $\epsilon_{T}, \epsilon_{L}$ and $\epsilon_{G}$, for each operator involved. They are not, however, arbitrary. Suppose we choose $\epsilon_{T}=\epsilon$ as our benchmark. This parameter regularizes the operator $\Delta_{T}$ or, if $a=1$, the 
operator $\Delta_{1}$. In flat space $\Delta_{1}=-\partial^{2}$ is just a diagonal product of two derivatives. Then, in order to regularize an operator $-a^{k} \partial^{2}$ we have to use the regulator $\epsilon / a^{k / 2}$. Thus, we conclude that we have to choose

$$
\epsilon_{T}=\epsilon, \epsilon_{L}^{2}=\epsilon^{2} / a, \epsilon_{G}^{2}=\epsilon^{2} / \sqrt{a} .
$$

This choice, as we will see in a moment, makes the regularization procedure gauge independent. The relation between $\epsilon_{T}$ and $\epsilon_{L}$ is consistent with the analysis made by Kabat [12] in two dimensions on the basis of the BRST invariance.

We note that (6.2) includes the relation (5.7) which we derived on the basis of the formal arguments. The opposite however is not true. That is why (5.7) alone is not enough to remove all dependence on $a$ while this can be done using (6.2). With the choice (6.2) for the regulators the effective action (6.1) becomes completely independent of the gauge fixing parameter $a$ and, moreover, it is the same as in the minimal gauge $a=1$,

$$
W_{\text {Maxwell }}=-\frac{1}{2} \int_{\epsilon^{2}}^{\infty} \frac{d s}{s}\left(\operatorname{Tr} e^{-s \Delta_{1}}-2 \operatorname{Tr} e^{+s \square_{0}}\right) .
$$

We note that if there exist some zero modes of the operators in question the discussion should be supplemented by subtraction of the contribution of these zero modes (see [19] and [21] for a discussion in two dimensions). This subtraction modifies the logarithmic UV divergent terms in the action.

\section{Cosmological and Newton's constants}

Using the heat kernel coefficient (4.8) one calculates the power law divergences in the effective action (4.3) on the curved background,

$$
W_{\mathrm{eff}}=-\lambda(\epsilon) \int d^{d} x \sqrt{g}-\frac{1}{16 \pi G(\epsilon)} \int d^{d} x \sqrt{g} R,
$$

where

$$
\lambda(\epsilon)=-\frac{1}{d(4 \pi)^{d / 2}} \frac{1}{\epsilon^{d}}(d-2)
$$

is the induced cosmological constant and

$$
\frac{1}{16 \pi G(\epsilon)}=\frac{1}{(d-2)} \frac{1}{(4 \pi)^{d / 2}} \frac{1}{\epsilon^{d-2}}\left(\frac{1}{6}(d-2)-1\right)
$$

is the induced Newton's constant. We notice that $(d-2)=N_{s=1}(d)$ is the number of on-shell degrees of freedom of a spin $s=1$ particle in dimension $d$.

\section{Minkowski spacetime: heat kernel in momentum space}

In Minkowski spacetime one can use the representation of the heat kernel by means of the Fourier transform. For the heat kernel of the nonminimal operator (2.3) one has that

$$
K_{\nu}^{\mu}\left(s, x, x^{\prime}\right)=\frac{1}{(2 \pi)^{d}} \int d^{p} K_{\nu}^{\mu}(s, p) e^{-i p\left(x-x^{\prime}\right)},
$$


where $K_{\nu}^{\mu}(s, p)$ satisfies equation

$$
\partial_{s} K_{\nu}^{\mu}(s, p)+\left(p^{2} \delta_{\sigma}^{\mu}+(a-1) p^{\mu} p_{\sigma}\right) K_{\nu}^{\sigma}(s, p)=0
$$

and the initial condition $K_{\nu}^{\mu}(s=0, p)=\delta_{\nu}^{\mu}$. The solution is easily found,

$$
K_{\nu}^{\mu}(s, p)=\left(e^{-s \Delta_{a}}\right)_{\nu}^{\mu}=\left(\delta_{\nu}^{\mu}-\frac{p^{\mu} p_{\nu}}{p^{2}}\right) e^{-p^{2} s}+\frac{p^{\mu} p_{\nu}}{p^{2}} e^{-a p^{2} s} .
$$

This is exactly the form (4.5), where in the momentum space the projectors are defined as

$$
\mathcal{P}_{\nu}^{\mu}=\frac{p^{\mu} p_{\nu}}{p^{2}}, \Pi_{\nu}^{\mu}=\left(\delta_{\nu}^{\mu}-\frac{p^{\mu} p_{\nu}}{p^{2}}\right) .
$$

Correspondingly, for the heat kernel of the ghost operator we have that

$$
K^{G}(s, p)=e^{-s \sqrt{a} p^{2}} .
$$

\section{$9 \quad$ Entanglement entropy}

On a hypersurface of constant time in Minkowski spacetime we consider a co-dimension two surface $\Sigma$. Entanglement entropy is defined by tracing over the modes which reside inside the surface. Even if one starts with a pure quantum state after the tracing over one ends up with a mixed state characterized by a density matrix. The corresponding entropy is called entanglement entropy. In oder to calculate the entanglement entropy one usually uses the so-called replica trick. It consists in introducing a small angle deficit $\delta=2 \pi(1-\alpha)$ at the surface and differentiating the effective action computed on this conical space with respect to the angle deficit (see [4] for a review). This procedure may be complicated in general. In some simple cases, when, for example, the surface $\Sigma$ is infinite $(d-2)$-plane, the procedure is rather straightforward. First, we need to compute the trace of the heat kernel on the conical space. In the momentum space representation of the heat kernel this procedure was carried out in general in [8]. The result can be formulated as follows

$$
\operatorname{Tr} K_{\alpha}(s)=\frac{1}{(4 \pi)^{d / 2}}\left(\alpha V P_{d}(s)+\frac{\pi}{3 \alpha}\left(1-\alpha^{2}\right) A(\Sigma) P_{d-2}(s)\right)
$$

where we introduced

$$
P_{n}(s)=\frac{2}{\Gamma\left(\frac{n}{2}\right)} \int_{0}^{\infty} d p p^{n-1} \operatorname{Tr} K(s, p),
$$

$K(s, p)$ is the Fourier transform of the heat kernel in question. The normalization is chosen in such a way that for $\operatorname{Tr} K(s, p)=e^{-s p^{2}}$ we have that $P_{n}(s)=s^{-n / 2} \cdot A(\Sigma)$ is the area of the surface $\Sigma$. The second term in (9.1) contains a piece which is proportional to the angle deficit and, thus, contributes to the entropy. Entanglement entropy then takes the form [8]

$$
S=\frac{A(\Sigma)}{12(4 \pi)^{(d-2) / 2}} \int_{\epsilon^{2}}^{\infty} \frac{d s}{s} P_{d-2}(s) .
$$


Applying this general formula to the Maxwell theory with a generic gauge fixing term we have to consider separately the contributions of transverse, longitudinal and ghost modes:

$$
\begin{aligned}
P_{d-2}^{(T, L, G)}(s) & =\frac{2}{\Gamma\left(\frac{d-2}{2}\right)} \int_{0}^{\infty} d p p^{d-3} \operatorname{Tr} K^{(T, L, G)}(s, p), \\
\operatorname{Tr} K^{T}(s, p) & =\operatorname{Tr} e^{-s p^{2}} \Pi, \operatorname{Tr} K^{L}(s, p)=\operatorname{Tr} e^{-a s p^{2}} \mathcal{P}, \operatorname{Tr} K^{G}(s, p)=e^{-\sqrt{a} s p^{2}} .
\end{aligned}
$$

Since $\operatorname{Tr} \Pi=(d-1)$ and $\operatorname{Tr} \mathcal{P}=1$ we find

$$
P_{d-2}^{T}(s)=(d-1) s^{-(d-2) / 2}, P_{d-2}^{L}(s)=(a s)^{-(d-2) / 2}, P_{d-2}^{G}(s)=(\sqrt{a} s)^{-(d-2) / 2} .
$$

The entanglement entropy is sum of the contributions of each sector of the Maxwell theory,

$$
S=\frac{A(\Sigma)}{12(4 \pi)^{(d-2) / 2}}\left(\int_{\epsilon_{T}^{2}}^{\infty} \frac{d s}{s} P_{d-2}^{T}(s)+\int_{\epsilon_{L}^{2}}^{\infty} \frac{d s}{s} P_{d-2}^{L}(s)-2 \int_{\epsilon_{G}^{2}}^{\infty} \frac{d s}{s} P_{d-2}^{G}(s)\right)
$$

where the ghost modes give a negative contribution and each of three sectors (transverse, longitudinal and ghost) has its own regularization parameter as in the case of the effective action. For the choice (6.2) of the regulators the entanglement entropy clearly does not depend on the gauge fixing parameter $a$ and it equals

$$
S_{\mathrm{ent}}=\frac{A(\Sigma)}{6(d-2)(4 \pi)^{\frac{d-2}{2}} \epsilon^{d-2}}(d-2) .
$$

As before, we notice that $(d-2)=N_{s=1}(d)$ is the number of the on-shell degrees of freedom of a spin $s=1$ particle in $d$ dimensions.

We conclude that the prescription (6.2), which makes the effective action independent of the gauge fixing parameter $a$, automatically leads to the entanglement entropy which does not depend on $a$. This adds yet another aspect to the correspondence between the effective action and the entropy earlier studied in [7]. Our analysis in this section has been made in Minkowski spacetime. However, the result for the entanglement entropy in flat spacetime always gives the the leading UV divergent contribution (proportional to the area) to the entanglement entropy in curved spacetime. Thus, the entropy (9.7) shows the area law for the Maxwell theory in a curved spacetime.

\section{Kabat's contact term}

Black holes in a theory of gravity described by the gravitational action (7.1) have the Bekenstein-Hawking entropy proportional to the horizon area

$$
S_{\mathrm{BH}}=\frac{A}{4 \pi G(\epsilon)},
$$

measured in the units set by $G(\epsilon)$, the induced Newton's constant (7.3). This should be compared to the entanglement entropy of the horizon that we calculated in section 9 . Here we note that for all known matter fields, bosonic or fermionic, minimally coupled to 
gravity these two entropies are identical $[4,7]$. The difference, however, takes place for those fields that nonminimally couple to the background metric, i.e. couple directly to the curvature. In particular, this is so for the gauge fields as can be seen from the form of the field equation (2.3). The difference between the two entropies can be presented as follows

$$
S_{\mathrm{BH}}=S_{\mathrm{ent}}-Q,
$$

where

$$
Q=\frac{A(\Sigma)}{(d-2)(4 \pi)^{\frac{d-2}{2}} \epsilon^{d-2}}
$$

is what in the literature known as Kabat's contact term (the interest to this term has been recently renewed, see [20-22]). It appears due to a direct interaction of the gauge field with the tip of the cone. For a nonminimally coupled scalar field, where a similar phenomenon takes place, this was analyzed in [23]. The Ricci tensor on a conical space has a delta-like contribution from the conical singularity [24]

$$
R_{\mu \nu}=2 \pi(1-\alpha)\left(n_{\mu} n_{\nu}\right) \delta_{\Sigma}+R_{\mu \nu}^{r e g}
$$

where $n_{\mu}^{a}, a=1,2$ is a pair of vectors orthogonal to $\Sigma,\left(n_{\mu} n_{\nu}\right)=\sum_{a} n_{\mu}^{a} n_{\nu}^{a}$. The black hole entropy (10.1) is obtained as response of the gravitational action to a small angle deficit at the horizon, so that $Q$ arises as such a response which is due to the coupling of the vector field directly to the Ricci tensor,

$$
Q=\left.2 \pi\left\langle A^{\mu} A^{\nu}\right\rangle\left(n_{\mu} n_{\nu}\right)\right|_{\Sigma}
$$

In the context of the induced gravity a similar quantity, due to scalar fields, was introduced in [10]. This quantity does not have a statistical meaning and it just restores the balance between the entanglement entropy which has this meaning and the black hole (or conical) entropy [4]. Our main observation here is the following. Both the black hole entropy (10.1) and the entanglement entropy (9.7), as we have just shown, do not depend on the gauge fixing parameter $a$. Same is true for their difference $Q$ (10.3), (10.5). Thus, the contact term $Q$ appears to be gauge independent, provided the gauge invariant regularization is used as we explained in section 6. The gauge independence of $Q$ is not obvious if we use the representation (10.5). The other piece of evidence that the contact terms produce the gauge invariant effects is the observation made in [22] that they make observable and gauge invariant contribution to the force between two cosmic strings.

The quantity $Q$ (10.5) and the entanglement entropy (9.7) can be defined for any codimension two surface $\Sigma$. However, the black hole entropy (10.1) is defined only if $\Sigma$ is the bifurcation point of a Killing horizon. Thus, only in this case our analysis indicates that the contact term $Q$ is a gauge-independent quantity. We also note that only the leading UV divergence (for entanglement entropy correctly reproduced by calculation in Minkowski spacetime but generically valid for any type of horizon) is discussed in this section. 


\section{Conclusions}

As our final remark we note that the property that the effective action and the entanglement entropy do not depend on the gauge fixing parameter $a$ is not completely equivalent to the gauge invariance of the effective action or the entropy. The two, however, are based on the use of Ward's identities. That is why, although more work is likely needed to demonstrate, in full, the gauge invariance of the contact term $Q$, the fact that $Q$ does not depend on the gauge fixing parameter is a clear argument in favor of the gauge invariance of $Q$. The other evidence, as we already said, comes from the analysis made in [22]. The gauge invariance of $Q$, if definitely established, should shed more light on the nature of this term and on its relevance to the problem of the black hole entropy.

\section{Acknowledgments}

I thank W. Donnelly, T. Jacobson, D. Kabat, A. Wall and especially A. Barvinsky for discussions and inspiring remarks. It is my pleasure to thank the Kavli Institute for Theoretical Physics (KITP) at UC Santa Barbara for hospitality during the initial stage of this project. This research was supported in part by the National Science Foundation under Grant No. PHY11-25915. The email exchange and the useful remarks by C. Pope, E. Sezgin, M. Perry and R. Percacci are highly appreciated.

Open Access. This article is distributed under the terms of the Creative Commons Attribution License which permits any use, distribution and reproduction in any medium, provided the original author(s) and source are credited.

\section{References}

[1] A. Barvinsky and G. Vilkovisky, The generalized Schwinger-Dewitt technique in gauge theories and quantum gravity, Phys. Rept. 119 (1985) 1 [INSPIRE].

[2] T. Nishioka, S. Ryu and T. Takayanagi, Holographic entanglement entropy: an overview, J. Phys. A 42 (2009) 504008 [arXiv:0905.0932] [inSPIRE].

[3] H. Casini and M. Huerta, Entanglement entropy in free quantum field theory, J. Phys. A 42 (2009) 504007 [arXiv:0905.2562] [InSPIRE].

[4] S.N. Solodukhin, Entanglement entropy of black holes, Living Rev. Rel. 14 (2011) 8 [arXiv: 1104.3712] [INSPIRE].

[5] T. Jacobson, Gravitation and vacuum entanglement entropy, Int. J. Mod. Phys. D 21 (2012) 1242006 [arXiv:1204.6349] [INSPIRE].

[6] L. Susskind and J. Uglum, Black hole entropy in canonical quantum gravity and superstring theory, Phys. Rev. D 50 (1994) 2700 [hep-th/9401070] [INSPIRE].

[7] D.V. Fursaev and S.N. Solodukhin, On one loop renormalization of black hole entropy, Phys. Lett. B 365 (1996) 51 [hep-th/9412020] [INSPIRE].

[8] D. Nesterov and S.N. Solodukhin, Gravitational effective action and entanglement entropy in UV modified theories with and without Lorentz symmetry, Nucl. Phys. B 842 (2011) 141 [arXiv: 1007.1246] [INSPIRE]. 
[9] T. Jacobson, Black hole entropy and induced gravity, gr-qc/9404039 [INSPIRE].

[10] V.P. Frolov and D. Fursaev, Mechanism of generation of black hole entropy in Sakharov's induced gravity, Phys. Rev. D 56 (1997) 2212 [hep-th/9703178] [INSPIRE].

[11] A. Barvinsky, private communication.

[12] D.N. Kabat, Black hole entropy and entropy of entanglement, Nucl. Phys. B 453 (1995) 281 [hep-th/9503016] [INSPIRE].

[13] A. Sen, Logarithmic corrections to Schwarzschild and other non-extremal black hole entropy in different dimensions, arXiv:1205.0971 [INSPIRE].

[14] S. Bhattacharyya, B. Panda and A. Sen, Heat kernel expansion and extremal Kerr-Newmann black hole entropy in Einstein-Maxwell theory, JHEP 08 (2012) 084 [arXiv:1204.4061] [INSPIRE].

[15] A. Sen, Logarithmic corrections to rotating extremal black hole entropy in four and five dimensions, Gen. Rel. Grav. 44 (2012) 1947 [arXiv:1109.3706] [INSPIRE].

[16] A. Sen, Logarithmic corrections to $N=2$ black hole entropy: an infrared window into the microstates, arXiv:1108.3842 [INSPIRE].

[17] P.P. Gilkey, T.P. Branson and S.A. Fulling, Heat equation asymptotics of "nonminimal" operators on differential forms, J. Math. Phys. 32 (1991) 2089.

[18] R. Endo, Gauge dependence of the gravitational conformal anomaly for the electromagnetic field, Prog. Theor. Phys. 71 (1984) 1366 [INSPIRE].

[19] A. Barvinsky and S. Solodukhin, Nonminimal coupling, boundary terms and renormalization of the Einstein-Hilbert action and black hole entropy, Nucl. Phys. B 479 (1996) 305 [gr-qc/9512047] [INSPIRE].

[20] A.R. Zhitnitsky, Entropy, contact interaction with horizon and dark energy, Phys. Rev. D 84 (2011) 124008 [arXiv:1105.6088] [InSPIRE].

[21] W. Donnelly, A.C. Wall and A.C. Wall, Do gauge fields really contribute negatively to black hole entropy?, Phys. Rev. D 86 (2012) 064042 [arXiv:1206.5831] [INSPIRE].

[22] D. Kabat, D. Sarkar and D. Sarkar, Cosmic string interactions induced by gauge and scalar fields, Phys. Rev. D 86 (2012) 084021 [arXiv:1206.5642] [INSPIRE].

[23] S.N. Solodukhin, One loop renormalization of black hole entropy due to nonminimally coupled matter, Phys. Rev. D 52 (1995) 7046 [hep-th/9504022] [INSPIRE].

[24] D.V. Fursaev and S.N. Solodukhin, On the description of the Riemannian geometry in the presence of conical defects, Phys. Rev. D 52 (1995) 2133 [hep-th/9501127] [INSPIRE]. 